\title{
21st Century Research in Pediatric Psychiatry
}

\author{
PIERRE GRESSENS AND DONNA M. FERRIERO
}

Inserm, U676 [P.G.], Hôpital Robert Debré, F-75019 Paris, France; Institute for Reproductive and Developmental Biology [P.G.], Imperial College, Hammersmith Campus, London W12 ONN, United Kingdom; Departments of Pediatrics and Neurology [D.M.F.], University of California, San Francisco, California 94143

$\mathrm{T}$ he field of Pediatric Psychiatry encompasses many types of diseases and pathological conditions. These disorders affect a large number of children and represent a tremendous human, emotional, social, and economic burden for our families and society $(1,2)$. The progressive decrypting of the pathophysiology of psychiatric disorders has led to the blurring of the frontiers between pediatric psychiatry, child neurology, developmental neurobiology, cognitive neurosciences, and social sciences. Pediatric psychiatry has become a key component in the neuroscience field.

Over the past decade, there has been an increasing awareness of these diseases and better tools for diagnosis, which have contributed to enhance recognition of children with psychiatric disorders (3). This observation is true in all societies and cultures (4). In addition, the incidence of some psychiatric disorders, like autism, may be increasing due to the exposure to some environmental factors, although these issues remain a matter of debate (5).

An important genetic component has been identified for many of pediatric psychiatric disorders (6). However, for several diseases, environmental factors seem to also play a key role, and the interplay between these environmental factors and genetic factors, probably through epigenetic mechanisms, is probably crucial during brain development, leading to altered programs of neuronal differentiation and connectivity (7).

Despite the relative small number of laboratories and teams involved in the field of pediatric psychiatry, tremendous progress have been made, mainly because of the coalescence of

Received March 7, 2011; accepted March 10, 2011.

Correspondence: Pierre Gressens, M.D., Ph.D., Inserm U676, Hôpital Robert Debré, 48 Blvd Serurier, F-75019 Paris, France; e-mail: pierre.gressens@inserm.fr four different factors: i) new clinical and imaging tools have been developed for an early and specific diagnosis and for a refined phenotyping of homogenous subgroups of patients (endophenotypes) (8); ii) this, in combination with the major methodological advances in the genetic field has led to the identification of several genes, many of them being involved in brain development (9); iii) well-conducted epidemiological studies have demonstrated a strong association between prenatal and perinatal factors and the occurrence of several psychiatric disorders; iv) altogether, this has led to the development of original and relevant animal models of several pediatric psychiatric disorders, which have enabled investigators to dissect out the underlying cellular and molecular mechanisms (10).

Major contributors in the field of pediatric psychiatry have provided state-of-the-art reviews on some of the hottest topics in this rapidly moving field. Although this Special Issue is not exhaustive in this regard, it will cover several aspects and provide the reader a large overview of the recent and very exciting progress that this field has made in the recent past and will likely make in the future.

The first set of articles (11-14) reviews the evidence of the impact of prenatal and perinatal factors, including exposure to maternal depression or anxiety, drugs for these conditions, prematurity, glucocorticoids, and inflammation, on the development of childhood psychiatric disorders. The authors summarize the rapidly expanding literature on the topic and come up with original and exciting hypotheses, which could be tested in the near future in preclinical and clinical research settings.

Another important set of articles (10,15-18) focus on autism and covers several areas: i) modeling features of autism in animals; ii) the potential role of mitochondrial dysfunction in the diverse medical signs of autism; iii) sensory 
processing in autism; iv) quantitative nature of social impairment; and v) imaging abnormalities.

Two articles are dealing with the neurobiology of attention deficit/hyperactivity disorder (19) and of anxiety disorders (20), respectively. The impact of genetics on social cognition is also reviewed (21) as well as the role of epigenetic mechanisms in neurodevelopment (22). Finally, one article reviews the interplay between different psychiatric disorders and the Theory of the Mind (23).

Taken together, these outstanding reviews highlight the exciting advances in our understanding of complex neuropsychiatric disorders of childhood and will provide the reader with provocative ideas for further research and discussion. The better understanding of psychiatric disorders will lead, in the future, to better and earlier diagnosis and to the discovery and implementing of new treatments and prevention strategies.

\section{REFERENCES}

1. Ramsawh HJ, Chavira DA, Stein MB 2010 Burden of anxiety disorders in pediatric medical settings: prevalence, phenomenology, and a research agenda. Arch Pediatr Adolesc Med 164:965-972

2. Hsia RY, Belfer ML 2008 A framework for the economicanalysis of child and adolescent mental disorders. Int Rev Psychiatry 20:251-259

3. Willcutt EG, Pennington BF, Duncan L, Smith SD, Keenan JM, Wadsworth S, Defries JC, Olson RK 2010 Understanding the complex etiologies of developmental disorders: behavioral and molecular genetic approaches. J Dev Behav Pediatr 31:533-544

4. Srinath S, Kandasamy P, Golhar TS 2010 Epidemiology of child and adolescent mental health disorders in Asia. Curr Opin Psychiatry 23:330-336
5. Currenti SA 2010 Understanding and determining the etiology of autism. Cell Mol Neurobiol 30:161-171

6. State MW 2010 The genetics of child psychiatric disorders: focus on autism and Tourette syndrome. Neuron 68:254-269

7. Grafodatskaya D, Chung B, Szatmari P, Weksberg R 2010 Autism spectrum disorders and epigenetics. J Am Acad Child Adolesc Psychiatry 49:794-809

8. Levy Y, Ebstein RP 2009 Research review: crossing syndrome boundaries in the search for brain endophenotypes. J Child Psychol Psychiatry 50:657-668

9. Gerretsen P, Muller DJ, Tiwari A, Mamo D, Pollock BG 2009 The intersection of pharmacology, imaging, and genetics in the development of personalized medicine. Dialogues Clin Neurosci 11:363-376

10. Patterson PH 2011 Modeling autistic features in animals. Pediatr Res 69:34R-40R

11. Monk C, Fitelson EM, Werner E 2011 Mood disorders \& their pharmacological treatment during pregnancy: Is the future child affected? Pediatr Res 69:3R-10R

12. Johnson S, Marlow N 2011 Preterm birth and childhood psychiatric disorders. Pediatr Res 69:11R-18R

13. Huang LT 2011 The link between perinatal glucocorticoids exposure and psychiatric disorders. Pediatr Res 69:19R-25R

14. Meyer U, Feldon J, Dammann O 2011 Schizophrenia and autism: both shared and disorder-specific pathogenesis via perinatal inflammation? Pediatr Res 69:26R-33R

15. Frye RE, Rossignol DA 2011 Mitochondrial dysfunction can connect the diverse medical symptoms associated with autism spectrum disorders. Pediatr Res 69:41R-47R

16. Marco EJ 2011 Sensory Processing in Autism: A Review of Neurophysiologic Findings. Pediatr Res 69:48R-54R

17. Constantino JN 2011 The quantitative nature of autistic social impairment. Pediatr Res 69:55R-62R

18. Chen R, Jiao Y, Herskovits EH 2011 Structural MRI in autism spectrum disorder. Pediatr Res 69:63R-68R

19. Purper-Ouakil D, Ramoz N, Lepagnol-Bestel AM, Gorwood P, Simonneau M 2011 Neurobiology of attention deficit/hyperactivity disorder. Pediatr Res 69:69R-76R

20. Lampis V, Maziade M, Battaglia M. Animal models of human anxiety disorders: reappraisal from a developmental psychopathology vantage point. Pediatr Res 69:77R-84R

21. Skuse DF, Gallagher L 2011 Genetic influences on social cognition. Pediatr Res 69:85R-91R

22. Zahir FR, Brown CJ 2011 Epigenetic impacts on neurodevelopment: pathophysiological mechanisms and genetic modes of action. Pediatr Res 69:92R-100R

23. Korkmaz B 2011 Theory of mind (TOM) and neurodevelopmental disorders of childhood. Pediatr Res 69:101R-108R 